\title{
Unified contraction algorithm for multi-baryon correlators on the lattice
}

\author{
Takumi Doi*, Michael G. Endres \\ Theoretical Research Division, Nishina Center, RIKEN, Wako 351-0198, Japan
}

\begin{abstract}
We propose a novel algorithm for calculating multi-baryon correlation functions on the lattice. By considering the permutation of quarks (Wick contractions) and color/spinor contractions simultaneously, we construct a unified index list for the contraction where the redundancies in the original contraction are eliminated. We find that a significant reduction in the computational cost of correlators is achieved, e.g., by a factor of 192 for ${ }^{3} \mathrm{H}$ and ${ }^{3} \mathrm{He}$ nuclei, and a factor of 20736 for the ${ }^{4} \mathrm{He}$ nucleus, without assuming isospin symmetry. A further reduction is possible by exploiting isospin symmetry, and/or interchange symmetries associated with sink baryons, if such symmetries exist. Extensions for systems with hyperons are presented as well.
\end{abstract}

Keywords: Lattice QCD, Hadron-Hadron Interactions, Multi-Baryon Correlators, Contraction Algorithms

\section{Introduction}

Correlation functions of multi-baryon systems are the central quantities to be calculated when determining the properties and interactions of atomic nuclei directly from lattice QCD (+QED) simulations. The computational cost of constructing such correlators is, however, known to be exceptionally enormous for large mass number $A$, and one of the greatest challenges is to find an efficient algorithm for reducing it. The reason for such a high cost is that (i) the number of quark permutations (Wick contractions) grows factorially with $A$ and (ii) the contraction of color/spinor degrees of freedom (DoF)

\footnotetext{
${ }^{*}$ Corresponding author

Email address: doi@ribf.riken.jp (Takumi Doi)
} 
becomes exponentially large for large $A$. While there has been significant progress toward reducing this computational cost [1, 2, 3, 4, 4, 5], it continues to remain the most time-consuming part of the calculation, particularly for $A>21$

Lattice QCD simulations for multi-baryon systems date back to Refs. [13, 14], where energies of two-nucleon $(2 \mathrm{~N})$ systems in a box were extracted from temporal correlators in Euclidean space-time, and then related to $2 \mathrm{~N}$ scattering lengths through the use of Lüscher's formula [15, 16, 17]. Similar methods have been employed in recent studies as well [11, 18]. In Refs. [1, 2, 3], a new approach had been proposed, where nuclear forces were directly extracted from Nambu-Bethe-Salpeter (NBS) wave functions, or spacial correlators of $2 \mathrm{~N}$ systems. This method was successfully extended to general hadron-hadron interactions such as hyperon-nucleon (YN) and hyperon-hyperon (YY) potentials [19, 20, 21, 22, 23, 24, 25, 26]. A further extension was proposed in Ref. [12], where both spacial and temporal dependencies of correlators are utilized to extract non-local hadron-hadron potentials without requiring ground state saturation.

Only quite recently, however, have lattice QCD studies for three- and higher-baryon systems been initiated: the NPLQCD Collaboration demonstrated a feasibility study for the energy of a system with $\Xi^{0} \Xi^{0} n$ quantum numbers [10]; the PACS-CS Collaboration studied the energies of ${ }^{3} \mathrm{He}$ and ${ }^{4} \mathrm{He}$ at several lattice volumes and concluded that both ${ }^{3} \mathrm{He}$ and ${ }^{4} \mathrm{He}$ are bound states [4]. In Ref. [5], the HAL QCD Collaboration investigated threenucleon forces $(3 \mathrm{NF})$ using the NBS wave function of three nucleons, and repulsive $3 \mathrm{NF}$ at short distance were found in the triton $\left({ }^{3} \mathrm{H}\right)$ channel. One of the major obstacles in each of these studies was the computational cost of quark contractions, as discussed above.

The purpose of this paper is to present a novel algorithm for the computation of multi-baryon correlators. In particular, by considering the quark permutation and the color/spinor contractions simultaneously, we show that there exist large redundancies in the contributions to the correlator. By con-

\footnotetext{
${ }^{1}$ In this paper, we consider the computational cost of constructing multi-baryon correlators for a fixed ensemble size, neglecting the potential computational difficulty of achieving an acceptable signal/noise ratio for such correlators at late times. This unrelated issue, known as the signal/noise problem, grows exponentially with both mass number and time separation of correlators [6]. For various attempts to ameliorate this problem, see, e.g., Refs. [7, 8, 9, 10, 11, 12].
} 
structing a unified index list of non-vanishing contributions to the contraction with those redundancies eliminated, we can achieve a significant speedup for the computation of correlators. As will be described later, it is not necessary to assume a symmetry between different flavors (e.g., isospin symmetry) in this algorithm, although by doing so, an additional reduction in cost can be achieved.

This paper is organized as follows. In Section 2, we describe the multibaryon correlation functions under consideration and review the issues associated with computing contractions. In Section 3, we propose a new algorithm which utilizes a unified index list for evaluating contractions. In Section 4, the efficiency of the new algorithm is discussed, while Section 5 is devoted to summary and concluding remarks. Further details of our results are tabulated in Appendix A.

\section{Multi-baryon correlation functions}

We consider a $2 A$-point multi-baryon correlation function with a mass number $A$, defined by

$$
\begin{aligned}
& \Pi_{\alpha_{1}, \cdots, \alpha_{A} ; \alpha_{1}^{\prime}, \cdots, \alpha_{A}^{\prime}}\left(X_{1}, \cdots, X_{A} ; X_{1}^{\prime}, \cdots, X_{A}^{\prime}\right) \\
& \quad \equiv\left\langle B_{\alpha_{1}}\left(X_{1}\right) \cdots B_{\alpha_{A}}\left(X_{A}\right) \bar{B}_{\alpha_{A}^{\prime}}^{\prime}\left(X_{A}^{\prime}\right) \cdots \bar{B}_{\alpha_{1}^{\prime}}^{\prime}\left(X_{1}^{\prime}\right)\right\rangle,
\end{aligned}
$$

where $B_{\alpha_{i}}\left(\bar{B}_{\alpha_{i}^{\prime}}^{\prime}\right)$ denotes an appropriate baryon interpolating field in the sink (source) with a spinor index $\alpha_{i}\left(\alpha_{i}^{\prime}\right)$, and coordinate index $X_{i} \equiv\left(t_{i}, \vec{X}_{i}\right)\left(X_{i}^{\prime}\right)$. We consider a general baryon operator given by

$$
\begin{aligned}
B_{\alpha}(X) & =\epsilon_{c_{1} c_{2} c_{3}}\left(C \Gamma_{1}\right)_{\alpha_{1}, \alpha_{2}}\left(\Gamma_{2}\right)_{\alpha, \alpha_{3}} q\left(\xi_{1}\right) q\left(\xi_{2}\right) q\left(\xi_{3}\right), \\
\bar{B}_{\alpha^{\prime}}^{\prime}\left(X^{\prime}\right) & =\epsilon_{c_{1}^{\prime} c_{2}^{\prime} c_{3}^{\prime}}\left(C \Gamma_{1}^{\prime}\right)_{\alpha_{1}^{\prime}, \alpha_{2}^{\prime}}\left(\Gamma_{2}^{\prime}\right)_{\alpha^{\prime}, \alpha_{3}^{\prime}} \bar{q}\left(\xi_{3}^{\prime}\right) \bar{q}\left(\xi_{2}^{\prime}\right) \bar{q}\left(\xi_{1}^{\prime}\right),
\end{aligned}
$$

where $c_{i}\left(c_{i}^{\prime}\right)$ denotes color indices, and $\xi_{i}\left(\xi_{i}^{\prime}\right)$ is a symbolic label for the collection of indices $\left\{x_{i}, c_{i}, \alpha_{i}\right\}$ with $x_{i}$ being a quark coordinate index. Summation over repeated indices is implied. $C=\gamma_{4} \gamma_{2}$ is the charge conjugation matrix and $\Gamma_{i}\left(\Gamma_{i}^{\prime}\right)$ are appropriate $\gamma$-matrices. For instance, the choice of $\left(\Gamma_{1}, \Gamma_{2}\right)=\left(\Gamma_{1}^{\prime}, \Gamma_{2}^{\prime}\right)=\left(\gamma_{5}, 1\right)$ is often employed for an octet baryon field. In the case of point sources and point sinks for quark fields, $X=x_{1}=x_{2}=x_{3}$ and $X^{\prime}=x_{1}^{\prime}=x_{2}^{\prime}=x_{3}^{\prime}$. Generalization to smeared quark fields in the sink and/or source is straight-forward; in such cases the coordinate indices $x_{i}\left(x_{i}^{\prime}\right)$ are replaced by associated smearing parameters. 


\subsection{Computation using a straightforward algorithm}

As described in Sec.1, the computational cost of a multi-baryon correlator diverges quickly for large $A$. One can estimate the number of contractions in Eq. (1) for a given $\left\{\alpha_{i}, \alpha_{i}^{\prime}, t_{i}, t_{i}^{\prime}\right\}$ as follows. First, if we consider the 3-flavor space, the number of quark permutations (Wick contractions) amount to $N_{\text {perm }}=N_{u} ! \cdot N_{d} ! \cdot N_{s}$ ! where $N_{u}, N_{d}$ and $N_{s}$ are the number of up, down and strange quarks in the system, respectively. For instance, $N_{\text {perm }}=36$ for ${ }^{2} \mathrm{H}$, 2880 for ${ }^{3} \mathrm{H} /{ }^{3} \mathrm{He}$ and 518400 for ${ }^{4} \mathrm{He}$. Second, one must take into account the contractions for the color/spinor DoF. To carry out the counting, we exploit the sparse nature of $\gamma$-matrices and $\epsilon$-tensor: for each baryon in the sink or source, we attribute a factor of six to each color loop (i.e., sum over each color index), and a factor of four to each spinor loop (i.e., sum over each spinor index). The total cost of the color/spinor contractions therefore scale as $N_{\text {loop }}=6^{2 A} \cdot 4^{2 A}$. Particularly, we find $N_{\text {loop }}$ to be $\mathcal{O}\left(10^{5}\right)$ for ${ }^{2} \mathrm{H}, \mathcal{O}\left(10^{8}\right)$ for ${ }^{3} \mathrm{H} /{ }^{3} \mathrm{He}$ and $\mathcal{O}\left(10^{11}\right)$ for ${ }^{4} \mathrm{He}$. Third, we must repeat the above computation for all possible spacial variables at the sink, $\left\{\vec{X}_{1}, \cdots, \vec{X}_{A}\right\}$. For instance, when extracting the energy of the system, a zero-momentum projection for each baryon is commonly performed [4, 10, 11, 13, 14, 18]. When determining hadron-hadron potentials, NBS wave functions are extracted by imposing zero-momentum for the center of gravity, and the dependencies on relative coordinates between baryons are subject of interest [1, 2, 3, 5, 12, 19, 20, 21, $22,23,24,25,26$. In both cases, the computational cost will be multiplied by a factor of $N_{\mathrm{vol}}=L^{3 A}$, where $L$ is the spacial extent of the lattice 2

\subsection{Block algorithm}

Recently, algorithmic progress has been achieved for the computation of multi-baryon correlators [1, 2, 3, 4, 4, 5], by considering a block of three-quark propagators combined into a baryon sink. For simplicity, let us consider a $2 A$-point nucleon correlation function with $A=2$, given by

$$
\Pi_{\alpha, \beta ; \alpha^{\prime}, \beta^{\prime}}\left(X_{1}, X_{2} ; X_{1}^{\prime}, X_{2}^{\prime}\right)=\left\langle p_{\alpha}\left(X_{1}\right) n_{\beta}\left(X_{2}\right) \bar{n}_{\beta^{\prime}}^{\prime}\left(X_{2}^{\prime}\right) \bar{p}_{\alpha^{\prime}}^{\prime}\left(X_{1}^{\prime}\right)\right\rangle
$$

\footnotetext{
${ }^{2}$ One typically does not count the computational cost associated with summing over spacial baryon coordinates, $\left\{\vec{X}_{1}^{\prime}, \cdots, \vec{X}_{A}^{\prime}\right\}$, at the source. Rather, a sum over quark coordinate indices is implicitly performed at the source by solving quark propagators with smearing sources. If one uses, e.g., all-to-all propagators, an additional factor of $N_{\text {vol }}$ would arise, however.
} 
where the proton and neutron fields are defined by

$$
\begin{aligned}
p_{\alpha}(X) & =+\epsilon_{c_{1} c_{2} c_{3}}\left(C \Gamma_{1}^{p}\right)_{\alpha_{1}, \alpha_{2}}\left(\Gamma_{2}^{p}\right)_{\alpha, \alpha_{3}} u\left(\xi_{1}\right) d\left(\xi_{2}\right) u\left(\xi_{3}\right), \\
n_{\alpha}(X) & =-\epsilon_{c_{1} c_{2} c_{3}}\left(C \Gamma_{1}^{n}\right)_{\alpha_{1}, \alpha_{2}}\left(\Gamma_{2}^{n}\right)_{\alpha, \alpha_{3}} d\left(\xi_{1}\right) u\left(\xi_{2}\right) d\left(\xi_{3}\right), \\
\bar{p}_{\alpha^{\prime}}^{\prime}\left(X^{\prime}\right) & =+\epsilon_{c_{1}^{\prime} c_{2}^{\prime} c_{3}^{\prime}}\left(C \Gamma_{1}^{\prime p}\right)_{\alpha_{1}^{\prime}, \alpha_{2}^{\prime}}\left(\Gamma_{2}^{\prime p}\right)_{\alpha^{\prime}, \alpha_{3}^{\prime}} \bar{u}\left(\xi_{3}^{\prime}\right) \bar{d}\left(\xi_{2}^{\prime}\right) \bar{u}\left(\xi_{1}^{\prime}\right), \\
\bar{n}_{\alpha^{\prime}}^{\prime}\left(X^{\prime}\right) & =-\epsilon_{c_{1}^{\prime} c_{2}^{\prime} c_{3}^{\prime}}\left(C \Gamma_{1}^{\prime n}\right)_{\alpha_{1}^{\prime}, \alpha_{2}^{\prime}}\left(\Gamma_{2}^{\prime n}\right)_{\alpha^{\prime}, \alpha_{3}^{\prime}}^{\prime} \bar{d}\left(\xi_{3}^{\prime}\right) \bar{u}\left(\xi_{2}^{\prime}\right) \bar{d}\left(\xi_{1}^{\prime}\right) .
\end{aligned}
$$

We construct blocks of three-quark propagators defined by

$$
\begin{aligned}
f_{\alpha}^{p}(X ; & \left.\xi_{1}^{\prime}, \xi_{2}^{\prime}, \xi_{3}^{\prime}\right) \equiv\left\langle p_{\alpha}(X) \cdot \bar{u}\left(\xi_{3}^{\prime}\right) \bar{d}\left(\xi_{2}^{\prime}\right) \bar{u}\left(\xi_{1}^{\prime}\right)\right\rangle \\
= & \epsilon_{c_{1} c_{2} c_{3}}\left(C \Gamma_{1}^{p}\right)_{\alpha_{1}, \alpha_{2}}\left(\Gamma_{2}^{p}\right)_{\alpha, \alpha_{3}} \\
& \times\left[S_{u}\left(\xi_{1}, \xi_{1}^{\prime}\right) S_{u}\left(\xi_{3}, \xi_{3}^{\prime}\right)-S_{u}\left(\xi_{1}, \xi_{3}^{\prime}\right) S_{u}\left(\xi_{3}, \xi_{1}^{\prime}\right)\right] S_{d}\left(\xi_{2}, \xi_{2}^{\prime}\right)
\end{aligned}
$$

and

$$
\begin{aligned}
& f_{\beta}^{n}(X ;\left.\xi_{1}^{\prime}, \xi_{2}^{\prime}, \xi_{3}^{\prime}\right) \equiv-\left\langle n_{\beta}(X) \cdot \bar{d}\left(\xi_{3}^{\prime}\right) \bar{u}\left(\xi_{2}^{\prime}\right) \bar{d}\left(\xi_{1}^{\prime}\right)\right\rangle \\
&= \epsilon_{c_{1} c_{2} c_{3}}\left(C \Gamma_{1}^{n}\right)_{\alpha_{1}, \alpha_{2}}\left(\Gamma_{2}^{n}\right)_{\beta, \alpha_{3}} \\
& \quad \times\left[S_{d}\left(\xi_{1}, \xi_{1}^{\prime}\right) S_{d}\left(\xi_{3}, \xi_{3}^{\prime}\right)-S_{d}\left(\xi_{1}, \xi_{3}^{\prime}\right) S_{d}\left(\xi_{3}, \xi_{1}^{\prime}\right)\right] S_{u}\left(\xi_{2}, \xi_{2}^{\prime}\right),
\end{aligned}
$$

for all possible indices $\left\{X, \xi_{i}^{\prime}\right\}$, where $S_{q}\left(\xi_{i}, \xi_{j}^{\prime}\right) \equiv\left\langle q\left(\xi_{i}\right) \bar{q}\left(\xi_{j}^{\prime}\right)\right\rangle$ denotes a quark propagator associated with the flavor $q=\{u, d, s\}$. Using Eqs. (9) and (10), the correlation function can be written as

$$
\begin{aligned}
& \Pi_{\alpha, \beta ; \alpha^{\prime}, \beta^{\prime}}\left(X_{1}, X_{2} ; X_{1}^{\prime}, X_{2}^{\prime}\right) \\
& =\sum_{\sigma} f_{\alpha}^{p}\left(X_{1} ; \xi_{\sigma(1)}^{\prime}, \xi_{\sigma(2)}^{\prime}, \xi_{\sigma(3)}^{\prime}\right) \cdot f_{\beta}^{n}\left(X_{2} ; \xi_{\sigma(4)}^{\prime}, \xi_{\sigma(5)}^{\prime}, \xi_{\sigma(6)}^{\prime}\right) \\
& \quad \times \epsilon_{c_{1}^{\prime} c_{2}^{\prime} c_{3}^{\prime}}\left(C \Gamma_{1}^{\prime p}\right)_{\alpha_{1}^{\prime}, \alpha_{2}^{\prime}}\left(\Gamma_{2}^{\prime p}\right)_{\alpha^{\prime}, \alpha_{3}^{\prime}} \cdot \epsilon_{c_{4}^{\prime} c_{5}^{\prime} c_{6}^{\prime}}\left(C \Gamma_{1}^{\prime n}\right)_{\alpha_{4}^{\prime}, \alpha_{5}^{\prime}}\left(\Gamma_{2}^{\prime n}\right)_{\beta^{\prime}, \alpha_{6}^{\prime}} \cdot \operatorname{sign}(\sigma),(11)
\end{aligned}
$$

where $\sum_{\sigma} \equiv \sum_{\sigma_{u}} \sum_{\sigma_{d}}$ with $\sigma_{u}\left(\sigma_{d}\right)$ representing the permutation among up (down) quarks, and $\operatorname{sign}(\sigma)=\operatorname{sign}\left(\sigma_{u}\right) \operatorname{sign}\left(\sigma_{d}\right)$ representing a sign factor which arises from the anti-commuting property of fermions.

There are several significant advantages to using Eq. (11) over the straightforward approach. First, in terms of the permutation, we note that Eq. (9) is antisymmetric under the exchange of two up quarks in the proton. A similar property holds for Eq. (10) under the exchange of two down quarks in the neutron. Generally speaking, by exploiting these features, one can restrict the full permutation appearing in Eq. (11), which we refer to as $\sigma_{\text {full }}$, to a sub-permutation $\sigma_{\text {sub }}$, which excludes such exchanges, thus reducing $N_{\text {perm }}$ 
by a factor of $2^{A}[1,2,3,4,4]$. Second, one finds that since the color/spinor contractions in the sink are performed prior to evaluating Eq. (11), $N_{\text {loop }}$ is reduced from $6^{2 A} \cdot 4^{2 A}$ to $6^{A} \cdot 4^{A}$. Note that the computational cost of evaluating Eqs. (9) and (10) is negligible, once up/down quark propagators are determined. Finally, this algorithm enables us to reduce the computational cost for the momentum projection. In fact, it is efficient to transform $f_{\alpha}^{p}, f_{\beta}^{n}$ to momentum-space first, prior to the calculation of Eq. (11). In this way, (e.g., in the case of the computation of NBS wave functions), one can perform the zero-momentum projection onto the center of gravity utilizing the convolution technique. This reduces $N_{\text {vol }}$ from $L^{3 A}$ down to $\mathcal{O}\left(L^{3 A-3}\right)$ [2]. Furthermore, if one is interested in only the energy of the system using the correlator with each sink baryon projected onto zero-momentum (or any fixed momentum), $N_{\mathrm{vol}}=\mathcal{O}(1)$, insensitive to $A[4]$.

We note that an additional improvement has been carried out in Ref. [4], in the isospin symmetric limit. By exploiting the permutation symmetry of protons and neutrons in the baryon interpolating field as well as other techniques, they achieved a significant reduction of $N_{\text {perm }}$, down to $N_{\text {perm }}=$ 93 for ${ }^{3} \mathrm{He}\left({ }^{3} \mathrm{H}\right)$ and $N_{\text {perm }}=1107$ for ${ }^{4} \mathrm{He}[4]$.

\section{Unified contraction algorithm}

We develop a new technique for evaluating contractions in correlation functions such as those defined in Eq. (1) by considering the permutation of quarks (Wick contractions) and the color/spinor contractions simultaneously. In doing so, we may eliminate redundancies as much as possible. Although the technique is rather general, and may be applied as an extension of either the straightforward algorithm or block algorithm, we focus our study on the latter case for simplicity.

To demonstrate the idea, we again consider a $2 A$-point nucleon correlation function with $A=2$, represented by block components given in Eq. (11). In our algorithm, we evaluate Eq. (11) under the condition that quarks of the same flavor have the same space-time source point, or more generally, have the same space-time smearing function at the source. Under this condition, a permutation of quark operators in the source is equivalent to a permutation of color and spinor indices of the corresponding quark sources, and thus we can rewrite Eq. (11) as

$$
\begin{aligned}
& \Pi_{\alpha, \beta ; \alpha^{\prime}, \beta^{\prime}}\left(X_{1}, X_{2} ; X_{1}^{\prime}, X_{2}^{\prime}\right) \\
& \quad=f_{\alpha}^{p}\left(X_{1} ; \xi_{1}^{\prime}, \xi_{2}^{\prime}, \xi_{3}^{\prime}\right) \cdot f_{\beta}^{n}\left(X_{2} ; \xi_{4}^{\prime}, \xi_{5}^{\prime}, \xi_{6}^{\prime}\right) \times C_{\alpha^{\prime} \beta^{\prime}}^{p n}\left(\xi_{1}^{\prime}, \cdots, \xi_{6}^{\prime}\right),
\end{aligned}
$$


where

$$
\begin{aligned}
& C_{\alpha^{\prime} \beta^{\prime}}^{p n}\left(\xi_{1}^{\prime}, \cdots, \xi_{6}^{\prime}\right) \\
& \equiv \sum_{\sigma^{-1}} \epsilon_{c_{\sigma(1)}^{\prime} c_{\sigma(2)}^{\prime} c_{\sigma(3)}^{\prime}}\left(C \Gamma_{1}^{\prime p}\right)_{\alpha_{\sigma(1)}^{\prime}, \alpha_{\sigma(2)}^{\prime}}\left(\Gamma_{2}^{\prime p}\right)_{\alpha^{\prime}, \alpha_{\sigma(3)}^{\prime}} \\
& \quad \times \epsilon_{c_{\sigma(4)}^{\prime} c_{\sigma(5)}^{\prime} c_{\sigma(6)}^{\prime}}\left(C \Gamma_{1}^{\prime n}\right)_{\alpha_{\sigma(4)}^{\prime}, \alpha_{\sigma(5)}^{\prime}}\left(\Gamma_{2}^{\prime n}\right)_{\beta^{\prime}, \alpha_{\sigma(6)}^{\prime}} \cdot \operatorname{sign}(\sigma),
\end{aligned}
$$

and the sum is carried over the inverse permutations $\sigma^{-1}$. An essential feature of this result is that the computation of a permutation is absent in Eq. (12). To compensate for this, one must instead perform a permutation calculation to evaluate Eq. (13). Since the summand in Eq. (13) is independent of the gauge field, however, this calculation need only be carried out once, and independently of any lattice simulation. As was the case for Eq. (11), the permutation sum in Eq. (13) may be taken over either the full permutation $\left(\sigma_{\text {full }}\right)$ or over the sub-permutation $\left(\sigma_{\text {sub }}\right)$. Generally, there is no difference between summing over $\sigma^{-1}$ or $\sigma$ in Eq. (13) in the former case, but in the latter case there is a difference, depending on the particular sub-permutation chosen. Note that in Eq. (13), although $\xi_{i}^{\prime}$ depends on the quark coordinate index at the source, because of the same-source condition imposed on the quark fields, this index is irrelevant in its evaluation.

We note that the evaluation of Eq. (13) amounts to preparing a unified index list for Wick and color/spinor loop contractions in which only nonzero components of the coefficient matrix $C_{\alpha^{\prime} \beta^{\prime}}^{p n}\left(\xi_{1}^{\prime}, \cdots, \xi_{6}^{\prime}\right)$ are tabulated. In particular, if there exists any redundancy and/or cancellation among contributions in the original contraction, they are automatically consolidated when constructing the unified index list. Considering the sparse nature of $\gamma$-matrices and $\epsilon$-tensors together, it is expected that the number of nonzero elements in the coefficient matrix is rather small, resulting in significant speedups in the computation of correlators 3 Note also that it is unnecessary to assume any symmetry between different flavors (e.g., isospin symmetry) in this algorithm, since only permutations among quarks of the same flavor are utilized. Various techniques to improve the signal in correlation functions have been investigated, including the use of all-to-all propagators [28] and

\footnotetext{
${ }^{3}$ In evaluating multi-hadron correlation functions, correlation functions may suffer from round-off error due to a large number of cancellations among contributing terms [27]. By evaluating Eq. (13), a subset of these cancellations are performed exactly using integer arithmetic, resulting in a reduction in round-off errors.
} 
novel smearing methods such as distillation [29]. Generally, the application of our approach in such cases is straight-forward, although the degree of cancellation achieved in evaluating the analog of Eq. (13) could depend heavily on the details of the operator construction.

\section{Efficiency of the unified contraction algorithm}

In order to examine the efficiency of the unified contraction algorithm, we explicitly evaluate coefficient matrices for typical examples of interest. Particularly, we study systems composed of octet baryons. With regards to the explicit spinor structure of a baryon operator at the source, we consider two choices,

$$
\begin{aligned}
& \left(\Gamma_{1}^{\prime}, \Gamma_{2}^{\prime}\right)=\left(\gamma_{5}, 1\right) \\
& \left(\Gamma_{1}^{\prime}, \Gamma_{2}^{\prime}\right)=\left(\gamma_{5} P_{n r}, P_{n r}\right), \quad P_{n r} \equiv\left(1+\gamma_{4}\right) / 2
\end{aligned}
$$

For each multi-baryon correlator, we employ either Eq. (14) or (15) for all baryon operators at the source, and do not mix the two choices. The choice of Eq. (15) has been employed in recent multi-baryon studies [4, 5], because only the upper half components of Dirac spinors survive (working in the Dirac basis for $\gamma$-matrices), thus reducing the number of spinor loop contractions by a factor of $2^{A} 4$ Hereafter, we refer to Eqs. (14) and (15) as "standard" and "non-relativistic" operators, respectively. Note that the evaluation of Eq. (13) does not depend on the spinor structure of a baryon operator at the $\operatorname{sink},\left(\Gamma_{1}, \Gamma_{2}\right)$.

When evaluating Eq. (13), we may in principle consider two choices for the permutation, $\sigma_{\text {full }}$ and $\sigma_{\text {sub }}$, as discussed in Sec. 2.2 and Sec. 3. While $\sigma_{\text {sub }}$ is trivially a better choice in the block algorithm, $\sigma_{\text {full }}$ offers an advantage in the unified contraction scheme. In particular, note that the coefficient matrix obtained from $\sigma_{\text {full }}$ solely depends on the structure of the source operators, while, in the case of $\sigma_{\text {sub }}$, it also depends on the sink baryons implicitly through the definition of $\sigma_{\text {sub }}$. Therefore, when one considers coupled channel correlation functions where source baryons and sink baryons could be different, the contraction list obtained from $\sigma_{\text {full }}$ has broader utility.

\footnotetext{
${ }^{4}$ Note that although the operator has an apparent non-relativistic form, the states, which are dynamically generated on the lattice, are not subject to any non-relativistic approximation.
} 
When sink baryons in the correlator are specified, one may exploit any existing inner-quark exchange symmetries at the sink to explicitly constrain the sum over indices in Eq. (12), and thus further reduce the computational cost of its evaluation. For example, if a baryon block is antisymmetric under the exchange of two indices $\xi_{i}^{\prime}$ and $\xi_{j}^{\prime}$ for $i \neq j$, and the coefficient matrix is also antisymmetric under exchange of the same two indices (as guaranteed under the full permutation), then one may constrain the sums in Eq. (12) such that $\xi_{i}^{\prime}<\xi_{j}^{\prime}$. For instance, in the case of multi-nucleon systems, such considerations will result in a reduction of $2^{A}$ in total 5 Note that this is the same reduction factor that is achieved by using $\sigma_{\text {sub }}$ as opposed to $\sigma_{\text {full }}$ in the block algorithm.

We carry out the construction of the unified contraction list using supercomputers, since in the case of a mass number $A>2$, the computational cost is found to be quite large depending on the operators chosen, naively growing factorially in each quark number (this exponential growth in computational cost can be eliminated in some cases by exploiting Pauli exclusion, as will be discussed later on). It is, however, just a one-time investment, and we intend to make the lists publicly available for future use. We investigate the utility of our algorithm by considering two-octet baryon systems in the case of $A=2$, and multi-nucleon systems, i.e., ${ }^{3} \mathrm{H} /{ }^{3} \mathrm{He}$ and ${ }^{4} \mathrm{He}$, for $A=3,4$. For simplicity, we consider single channel systems in this study, while extension to coupled channel systems is straightforward. The computational cost of correlators using the unified contraction algorithm can be estimated by counting the number of non-zero elements, $N_{\text {list }}$, in the coefficient matrix under the full permutation. The total number of terms in the contraction will then be given by $N_{\text {contr }}=N_{\text {list }} / 2^{A}$ after exploiting the inner-quark exchange to explicitly constrain the sums in Eq. (12). The values we obtain for $N_{\text {list }}$ and $N_{\text {contr }}$ are compiled in Appendix A, together with $N_{\text {perm }}$ and $N_{\text {loop }}$ obtained in the block algorithms.

In order to make a comparison of methods easier, we present an effective number of permutations, defined by $N_{\text {perm }}^{\text {eff }} \equiv N_{\text {contr }} / N_{\text {loop }}$ where $N_{\text {loop }}$ is taken from the corresponding block algorithm. The efficiency of our approach in comparison with the block algorithm (i.e., the speed-up factor) is given by

\footnotetext{
${ }^{5}$ One can apply a similar procedure for the unified contraction list with $\sigma_{\text {sub }}$, but the computational cost in the evaluation of Eq. (12) is equivalent to the cost of the choice with $\sigma_{\text {full }}$, as is evident from the definition of Eq. (13).
} 
the ratio $\eta \equiv N_{\text {perm }} / N_{\text {perm }}^{\text {eff }}$, with a ratio larger than unity indicating an improvement. From the tables in Appendix A, one immediately observes that the unified contraction algorithm yields better efficiency for all multibaryon systems under consideration. What is particularly noteworthy is the gain for $A=3$ and 4 with non-relativistic operators. In the case of ${ }^{3} \mathrm{H} /{ }^{3} \mathrm{He}$, a factor of 192 improvement over the block algorithm is achieved, and in the case of ${ }^{4} \mathrm{He}$, a factor of 20736 improvement is achieved. We have checked that these improvement factors are nearly realized in the actual lattice simulation code, as well. We also observe significant improvements in the case of $A=2$. These improvements are useful for, e.g., coupled channel calculations for YN, YY interactions, where considerable computational cost would be required using the block algorithm [25].

It is in order that we remark on several aspects of the unified contraction algorithm results presented in Appendix A. First, a special property is observed for ${ }^{4} \mathrm{He}$ correlators when non-relativistic operators are considered. Specifically, one finds that $N_{\text {list }}=518400$ is exactly the same as $N_{\text {perm }}$ obtained in the block algorithm with $\sigma_{\text {full }}$. This can be understood intuitively, by noting that color/spinor DoF are completely saturated in ${ }^{4} \mathrm{He}$ when quark sources are taken to be equal. This is a simple statement that, for every baryon spin component, the ${ }^{4} \mathrm{He}$ analog of the coefficient matrix defined in Eq. (13) is proportional to the product of two epsilon tensors in $\xi_{i}^{\prime}$ (one for each flavor). Similar saturation is realized for ${ }^{8} \mathrm{Be}$, when we employ the operator which uses all four spinors of quarks. For larger A, such fermion saturation can be exploited to reduce the computational cost of evaluating the unified contraction list by noting that the coefficient matrix must be proportional to an epsilon tensor for a subset of indices corresponding to the fermions for which the color/spinor degrees of freedom are fully saturated.

Second, although we tabulated $N_{\text {contr }}$ for all possible (upper) baryon spin indices $\left(\alpha^{\prime}, \beta^{\prime}, \cdots\right)$ at the source, it is not always necessary to calculate the correlator for all of them. For instance, the correlator for ${ }^{4} \mathrm{He}$ with $\alpha^{\prime}=\beta^{\prime}$ or $\gamma^{\prime}=\delta^{\prime}$ should be trivially zero because of the anti-commuting property of source baryons. It is interesting that the unified contraction algorithm exposes this feature explicitly, as is evident from the tables in Appendix A. In the same way, the ${ }^{4} \mathrm{He}$ correlator with, e.g., $\left(\alpha^{\prime}, \beta^{\prime}, \gamma^{\prime}, \delta^{\prime}\right)$ and $\left(\beta^{\prime}, \alpha^{\prime}, \delta^{\prime}, \gamma^{\prime}\right)$ should be same, so one can save computational time by calculating only one of them. Third, by imposing additional constraints on baryon sink operators, further reduction in computational cost is possible. For instance, when we consider the correlation function with each sink baryon projected onto 
zero-momentum, one may exploit any existing exchange symmetry among the same baryons in the sink [4]. This leads to a further reduction of the computational cost, e.g., by a factor of two and four for ${ }^{3} \mathrm{H} /{ }^{3} \mathrm{He}$ and ${ }^{4} \mathrm{He}$, respectively. Depending of the system of concern, one may also exploit additional symmetries, if any, to skip the computation of redundant correlators. For example, if one assumes isospin symmetry, the computational cost of the above mentioned ${ }^{4} \mathrm{He}$ correlator can be reduced further by about a factor of two.

The proposed algorithm is rather general, and so it is possible to extend the technique to other systems of interest. An immediate application is to multi-hadron correlators including mesons, although for purely mesonic correlators, it remains to be determined whether the technique offers any advantage over the recursive approaches of Refs. [30, 31]. Furthermore, the method may be extended to various problems in quantum mechanics, where Slater determinants often appear as the subject of interest. If the coefficients of the Slater determinants have a sparse nature, a similar prescription may provide an efficient alternative.

Finally, let us discuss the limitations of the unified contraction algorithm. In order to satisfy the same-source condition described in Sec. 3, the number of quarks associated with each flavor must be less than or equal to 12 due to Pauli exclusion. Therefore, the maximum mass number allowed is $A_{\max }=8$ in 2-flavor space and $A_{\max }=12$ in 3-flavor space, respectively. We note, however, that even for a system with $A>A_{\max }$, the presented algorithm is expected to be efficient since it can reduce the computational cost corresponding to the subspace of permutations, which is spanned by imposing the same-source condition on as many quarks as possible. Further studies are currently underway.

In the limit of large $A$, suppressing the computation of Wick contractions in Eq. (11) becomes most important, since $N_{\text {perm }}$ grows factorially in quark number, whereas the others $\left(N_{\text {loop }}, N_{\text {vol }}\right)$ grow exponentially with quark number. In fact, such an algorithm was proposed in [32], where the Wick contractions in Eq. (11) are expressed in terms of a determinant of quark propagators. It is then essential to realize that the computational cost of the determinant of an $n \times n$ matrix can be reduced from $\mathcal{O}(n !)$ to $\mathcal{O}\left(n^{3}\right)$ by employing LUdecomposition. Unfortunately, this is not an efficient algorithm for light nuclei such as ${ }^{4} \mathrm{He}$, since $N_{\text {loop }}$ and/or $N_{\text {vol }}$ remain overwhelmingly large as discussed in Sec. 2.1. However, as the mass number $A$ grows, the determinant algorithm would presumably become a useful approach. 


\section{Summary}

We have proposed an efficient algorithm for calculating multi-baryon correlation functions on the lattice. By considering the permutation of quarks (Wick contractions) and the color/spinor contractions simultaneously, we have shown that there exist large redundancies in the original contraction. We have developed a method to construct a unified index list for the contraction in which the redundancies are eliminated. It is noted that an assumption on the symmetry between different flavors (e.g., isospin symmetry) is not required in this algorithm, although imposing such symmetries leads to further computational savings. Possible extensions of this algorithm have also been discussed.

In order to determine how efficient the algorithm is, we have investigated several typical examples of interest, namely, two-octet baryon systems, ${ }^{3} \mathrm{H}$, ${ }^{3} \mathrm{He}$ and ${ }^{4} \mathrm{He}$. We have found that a significant speedup is achieved in all cases, in particular, by a factor of 192 for ${ }^{3} \mathrm{H}$ and ${ }^{3} \mathrm{He}$ nuclei and a factor of 20736 for the ${ }^{4}$ He nucleus. For typical correlators of concern, where each nucleon is projected onto zero-momentum, further speedup can be achieved, e.g., by a factor of 2 and 4 for ${ }^{3} \mathrm{H} /{ }^{3} \mathrm{He}$ and ${ }^{4} \mathrm{He}$, respectively. This achievement takes a significant step towards the ultimate objective of studying nuclear physics from first principles lattice simulations of QCD (+ QED).

\section{Acknowledgments}

We thank Dr. H. Suzuki for fruitful discussions; T.D. also thanks colleagues in the HAL QCD Collaboration for helpful discussions. The numerical simulations have been performed on SR16000 at YITP in Kyoto University, Blue Gene/Q at KEK and FX10 at Tokyo University. M.G.E. is supported by the Foreign Postdoctoral Researcher Program at RIKEN. This research is supported in part by MEXT Grant-in-Aid for Young Scientists (B) (24740146 and 23740227), the Large Scale Simulation Program of KEK No.12-11 (FY2011-2012) and SPIRE (Strategic Program for Innovative REsearch).

\section{Appendix A. Computational cost of the unified contraction algo- rithm}

Here, we show the computational cost of the unified contraction algorithm for various multi-baryon correlators. In particular, we tabulate the 
number of non-zero entries $\left(N_{\text {list }}\right)$ appearing in the coefficient matrix, the total number of contractions $\left(N_{\text {contr }}\right)$, and the effective permutation number $\left(N_{\text {perm }}^{\text {eff }} \equiv N_{\text {contr }} / N_{\text {loop }}\right)$. As discussed in Sec. 3, the number of contractions required to compute the correlation function is given by $N_{\text {contr }}=N_{\text {list }} / 2^{A}$ in the case of nucleons. For comparison, we also provide $N_{\text {perm }}$ and $N_{\text {loop }}$ for the block algorithm. The increase in efficiency achieved by the unified contraction algorithm over the block algorithm is given by the ratio $\eta \equiv N_{\text {perm }} / N_{\text {perm }}^{\text {eff }}$, where $N_{\text {perm }}$ is the number of permutations required in the block algorithm using the optimal choice, $\sigma_{\text {sub }}$.

Tables are provided for single channel two-octet baryon systems, ${ }^{3} \mathrm{H} /{ }^{3} \mathrm{He}$ and ${ }^{4} \mathrm{He}$. For each baryon in the system, only the upper spinor components are considered, i.e., $\alpha_{i}^{\prime}=(0,1)$ for $i=1, \ldots, A$, since each baryon field is expected to couple strongly to a corresponding positive parity baryon. Because the system is symmetric under the flip of all spin indices, we only give results for $\alpha_{1}^{\prime}=0$. For baryon interpolating fields in the source, we consider both standard and non-relativistic operators, as described in Sec. 4 .

\section{Appendix A.1. Two-octet baryon systems}

We consider single channel systems of two-octet baryons without assuming flavor symmetry. The contraction list falls into three classes based on the flavor content of baryons: (i) $p p, n n, \Sigma^{+} \Sigma^{+}, \Sigma^{-} \Sigma^{-}, \Xi^{0} \Xi^{0}, \Xi^{-} \Xi^{-}$systems, (ii) pn, $\Sigma^{+} \Xi^{0}, \Sigma^{-} \Xi^{-}$systems and (iii) $p \Sigma^{+}, n \Sigma^{-}, \Xi^{0} \Xi^{-}$systems. The correlation function under consideration is given by Eq. (4), or an analog of it. 
Table A.1: pp, nn, $\Sigma^{+} \Sigma^{+}, \Sigma^{-} \Sigma^{-}, \Xi^{0} \Xi^{0}, \Xi^{-} \Xi^{-}$systems with the standard operators.

\begin{tabular}{c|ccc|ccc|c}
\hline \hline source spin & \multicolumn{3}{|c|}{ block algorithm } & \multicolumn{3}{c|}{ unified contraction algorithm } & efficiency \\
\hline$\left(\alpha^{\prime}, \beta^{\prime}\right)$ & $N_{\text {loop }}$ & $N_{\text {perm }}\left(\sigma_{\text {full }}\right)$ & $N_{\text {perm }}\left(\sigma_{\text {sub }}\right)$ & $N_{\text {list }}$ & $N_{\text {contr }}$ & $N_{\text {perm }}^{\text {eff }}$ & $\eta$ \\
\hline$(0,0)$ & 576 & 48 & 12 & 0 & 0 & 0 & - \\
$(0,1)$ & 576 & 48 & 12 & 11088 & 2772 & 4.8 & 2.5 \\
\hline \hline
\end{tabular}

Table A.2: Same as above, but with the non-relativistic operators.

\begin{tabular}{c|ccc|ccc|c}
\hline \hline source spin & \multicolumn{3}{|c|}{ block algorithm } & \multicolumn{3}{c|}{ unified contraction algorithm } & efficiency \\
\hline$\left(\alpha^{\prime}, \beta^{\prime}\right)$ & $N_{\text {loop }}$ & $N_{\text {perm }}\left(\sigma_{\text {full }}\right)$ & $N_{\text {perm }}\left(\sigma_{\text {sub }}\right)$ & $N_{\text {list }}$ & $N_{\text {contr }}$ & $N_{\text {perm }}$ & $\eta$ \\
\hline$(0,0)$ & 144 & 48 & 12 & 0 & 0 & 0 & - \\
$(0,1)$ & 144 & 48 & 12 & 1008 & 252 & 1.8 & 6.9 \\
\hline \hline
\end{tabular}

Table A.3: $p n, \Sigma^{+} \Xi^{0}, \Sigma^{-} \Xi^{-}$systems with the standard operators.

\begin{tabular}{c|ccc|ccc|c}
\hline \hline source spin & \multicolumn{3}{|c|}{ block algorithm } & \multicolumn{3}{c|}{ unified contraction algorithm } & efficiency \\
\hline$\left(\alpha^{\prime}, \beta^{\prime}\right)$ & $N_{\text {loop }}$ & $N_{\text {perm }}\left(\sigma_{\text {full }}\right)$ & $N_{\text {perm }}\left(\sigma_{\text {sub }}\right)$ & $N_{\text {list }}$ & $N_{\text {contr }}$ & $N_{\text {perm }}^{\text {eff }}$ & $\eta$ \\
\hline$(0,0)$ & 576 & 36 & 9 & 8316 & 2079 & 3.6 & 2.5 \\
$(0,1)$ & 576 & 36 & 9 & 9432 & 2358 & 4.1 & 2.2 \\
\hline \hline
\end{tabular}

Table A.4: Same as above, but with the non-relativistic operators.

\begin{tabular}{c|ccc|ccc|c}
\hline \hline source spin & \multicolumn{3}{|c|}{ block algorithm } & \multicolumn{3}{c|}{ unified contraction algorithm } & efficiency \\
\hline$\left(\alpha^{\prime}, \beta^{\prime}\right)$ & $N_{\text {loop }}$ & $N_{\text {perm }}\left(\sigma_{\text {full }}\right)$ & $N_{\text {perm }}\left(\sigma_{\text {sub }}\right)$ & $N_{\text {list }}$ & $N_{\text {contr }}$ & $N_{\text {perm }}^{\text {eff }}$ & $\eta$ \\
\hline$(0,0)$ & 144 & 36 & 9 & 756 & 189 & 1.3 & 6.9 \\
$(0,1)$ & 144 & 36 & 9 & 1008 & 252 & 1.8 & 5.1 \\
\hline \hline
\end{tabular}


Table A.5: $p \Sigma^{+}, n \Sigma^{-}, \Xi^{0} \Xi^{-}$systems with the standard operators.

\begin{tabular}{c|ccc|ccc|c}
\hline \hline source spin & \multicolumn{3}{|c|}{ block algorithm } & \multicolumn{3}{c|}{ unified contraction algorithm } & efficiency \\
\hline$\left(\alpha^{\prime}, \beta^{\prime}\right)$ & $N_{\text {loop }}$ & $N_{\text {perm }}\left(\sigma_{\text {full }}\right)$ & $N_{\text {perm }}\left(\sigma_{\text {sub }}\right)$ & $N_{\text {list }}$ & $N_{\text {contr }}$ & $N_{\text {perm }}$ & $\eta$ \\
\hline$(0,0)$ & 576 & 24 & 6 & 5400 & 1350 & 2.3 & 2.6 \\
$(0,1)$ & 576 & 24 & 6 & 7776 & 1944 & 3.4 & 1.8 \\
\hline \hline
\end{tabular}

Table A.6: Same as above, but with the non-relativistic operators.

\begin{tabular}{c|ccc|ccc|c}
\hline \hline source spin & \multicolumn{3}{|c|}{ block algorithm } & \multicolumn{3}{|c|}{ unified contraction algorithm } & efficiency \\
\hline$\left(\alpha^{\prime}, \beta^{\prime}\right)$ & $N_{\text {loop }}$ & $N_{\text {perm }}\left(\sigma_{\text {full }}\right)$ & $N_{\text {perm }}\left(\sigma_{\text {sub }}\right)$ & $N_{\text {list }}$ & $N_{\text {contr }}$ & $N_{\text {perm }}^{\text {eff }}$ & $\eta$ \\
\hline$(0,0)$ & 144 & 24 & 6 & 648 & 162 & 1.1 & 5.3 \\
$(0,1)$ & 144 & 24 & 6 & 864 & 216 & 1.5 & 4.0 \\
\hline \hline
\end{tabular}

Appendix A.2. Three-octet baryon systems

We consider the correlation function for ${ }^{3} \mathrm{H}$ given by

$$
\Pi_{\alpha \beta \gamma ; \alpha^{\prime} \beta^{\prime} \gamma^{\prime}} \equiv\left\langle p_{\alpha} n_{\beta} n_{\gamma} \bar{n}_{\gamma^{\prime}}^{\prime} \bar{n}_{\beta^{\prime}}^{\prime} \bar{p}_{\alpha^{\prime}}^{\prime}\right\rangle
$$

${ }^{3} \mathrm{He}$, and the hyperon analogs $\Sigma^{+} \Sigma^{+} \Xi^{0}, \Sigma^{-} \Sigma^{-} \Xi^{-}, \Sigma^{+} \Xi^{0} \Xi^{0}$, and $\Sigma^{-} \Xi^{-} \Xi^{-}$ share the same contraction list. 
Table A.7: ${ }^{3} \mathrm{H},{ }^{3} \mathrm{He}$ systems with the standard operators.

\begin{tabular}{c|ccc|ccc|c}
\hline \hline source spin & \multicolumn{3}{|c|}{ block algorithm } & \multicolumn{3}{c|}{ unified contraction algorithm } & efficiency \\
\hline$\left(\alpha^{\prime}, \beta^{\prime}, \gamma^{\prime}\right)$ & $N_{\text {loop }}$ & $N_{\text {perm }}\left(\sigma_{\text {full }}\right)$ & $N_{\text {perm }}\left(\sigma_{\text {sub }}\right)$ & $N_{\text {list }}$ & $N_{\text {contr }}$ & $N_{\text {perm }}^{\text {eff }}$ & $\eta$ \\
\hline$(0,0,0)$ & 13824 & 2880 & 360 & 0 & 0 & 0 & - \\
$(0,0,1)$ & 13824 & 2880 & 360 & 3775680 & 471960 & 34.1 & 10.5 \\
$(0,1,0)$ & 13824 & 2880 & 360 & 3775680 & 471960 & 34.1 & 10.5 \\
$(0,1,1)$ & 13824 & 2880 & 360 & 0 & 0 & 0 & - \\
\hline \hline
\end{tabular}

Table A.8: Same as above, but with the non-relativistic operators.

\begin{tabular}{c|ccc|ccc|c}
\hline \hline source spin & \multicolumn{3}{|c|}{ block algorithm } & \multicolumn{3}{c}{ unified contraction algorithm } & efficiency \\
\hline$\left(\alpha^{\prime}, \beta^{\prime}, \gamma^{\prime}\right)$ & $N_{\text {loop }}$ & $N_{\text {perm }}\left(\sigma_{\text {full }}\right)$ & $N_{\text {perm }}\left(\sigma_{\text {sub }}\right)$ & $N_{\text {list }}$ & $N_{\text {contr }}$ & $N_{\text {perm }}^{\text {eff }}$ & $\eta$ \\
\hline$(0,0,0)$ & 1728 & 2880 & 360 & 0 & 0 & 0 & - \\
$(0,0,1)$ & 1728 & 2880 & 360 & 25920 & 3240 & 1.9 & 192 \\
$(0,1,0)$ & 1728 & 2880 & 360 & 25920 & 3240 & 1.9 & 192 \\
$(0,1,1)$ & 1728 & 2880 & 360 & 0 & 0 & 0 & - \\
\hline \hline
\end{tabular}

Appendix A.3. Four-octet baryon systems

We consider the correlation function of ${ }^{4} \mathrm{He}$ given by

$$
\Pi_{\alpha \beta \gamma \delta ; \alpha^{\prime} \beta^{\prime} \gamma^{\prime} \delta^{\prime}} \equiv\left\langle p_{\alpha} p_{\beta} n_{\gamma} n_{\delta} \bar{n}_{\delta^{\prime}}^{\prime} \bar{n}_{\gamma^{\prime}}^{\prime} \bar{p}_{\beta^{\prime}}^{\prime} \bar{p}_{\alpha^{\prime}}^{\prime}\right\rangle
$$

The hyperon analogs $\Sigma^{+} \Sigma^{+} \Xi^{0} \Xi^{0}$ and $\Sigma^{-} \Sigma^{-} \Xi^{-} \Xi^{-}$share the same contraction list. 
Table A.9: ${ }^{4} \mathrm{He}$ system with the standard operators.

\begin{tabular}{c|ccc|ccc|c}
\hline \hline source spin & \multicolumn{3}{|c|}{ block algorithm } & \multicolumn{3}{c|}{ unified contraction algorithm } & efficiency \\
\hline$\left(\alpha^{\prime}, \beta^{\prime}, \gamma^{\prime}, \delta^{\prime}\right)$ & $N_{\text {loop }}$ & $N_{\text {perm }}\left(\sigma_{\text {full }}\right)$ & $N_{\text {perm }}\left(\sigma_{\text {sub }}\right)$ & $N_{\text {list }}$ & $N_{\text {contr }}$ & $N_{\text {perm }}^{\text {eff }}$ & $\eta$ \\
\hline$(0,0,0,0)$ & 331776 & 518400 & 32400 & 0 & 0 & 0 & - \\
$(0,0,0,1)$ & 331776 & 518400 & 32400 & 0 & 0 & 0 & - \\
$(0,0,1,0)$ & 331776 & 518400 & 32400 & 0 & 0 & 0 & - \\
$(0,0,1,1)$ & 331776 & 518400 & 32400 & 0 & 0 & 0 & - \\
$(0,1,0,0)$ & 331776 & 518400 & 32400 & 0 & 0 & 0 & - \\
$(0,1,0,1)$ & 331776 & 518400 & 32400 & 1407974400 & 87998400 & 265.2 & 122.2 \\
$(0,1,1,0)$ & 331776 & 518400 & 32400 & 1407974400 & 87998400 & 265.2 & 122.2 \\
$(0,1,1,1)$ & 331776 & 518400 & 32400 & 0 & 0 & 0 & - \\
\hline \hline
\end{tabular}

Table A.10: Same as above, but with the non-relativistic operators.

\begin{tabular}{c|ccc|ccc|c}
\hline \hline source spin & \multicolumn{3}{|c|}{ block algorithm } & \multicolumn{3}{|c|}{ unified contraction algorithm } & efficiency \\
\hline$\left(\alpha^{\prime}, \beta^{\prime}, \gamma^{\prime}, \delta^{\prime}\right)$ & $N_{\text {loop }}$ & $N_{\text {perm }}\left(\sigma_{\text {full }}\right)$ & $N_{\text {perm }}\left(\sigma_{\text {sub }}\right)$ & $N_{\text {list }}$ & $N_{\text {contr }}$ & $N_{\text {perm }}^{\text {eff }}$ & $\eta$ \\
\hline$(0,0,0,0)$ & 20736 & 518400 & 32400 & 0 & 0 & 0 & - \\
$(0,0,0,1)$ & 20736 & 518400 & 32400 & 0 & 0 & 0 & - \\
$(0,0,1,0)$ & 20736 & 518400 & 32400 & 0 & 0 & 0 & - \\
$(0,0,1,1)$ & 20736 & 518400 & 32400 & 0 & 0 & 0 & - \\
$(0,1,0,0)$ & 20736 & 518400 & 32400 & 0 & 0 & 0 & - \\
$(0,1,0,1)$ & 20736 & 518400 & 32400 & 518400 & 32400 & 1.6 & 20736 \\
$(0,1,1,0)$ & 20736 & 518400 & 32400 & 518400 & 32400 & 1.6 & 20736 \\
$(0,1,1,1)$ & 20736 & 518400 & 32400 & 0 & 0 & 0 & - \\
\hline \hline
\end{tabular}

\section{References}

[1] N. Ishii, S. Aoki and T. Hatsuda, Phys. Rev. Lett. 99, 022001 (2007) nucl-th/0611096.

[2] N. Ishii, S. Aoki and T. Hatsuda, PoS LATTICE 2008, 155 (2008) arXiv:0903.5497 [hep-lat]]. 
[3] S. Aoki, T. Hatsuda and N. Ishii, Prog. Theor. Phys. 123, 89 (2010) arXiv:0909.5585 [hep-lat]].

[4] T. Yamazaki, Y. Kuramashi and A. Ukawa, [PACS-CS Collaboration], Phys. Rev. D81, 111504 (2010). arXiv:0912.1383 [hep-lat]].

[5] T. Doi, et al. [HAL QCD Collaboration], Prog. Theor. Phys. 127, 723 (2012) arXiv:1106.2276 [hep-lat]].

[6] G. P. Lepage, in From Actions to Answers: Proceedings of the TASI 1989, edited by T. Degrand and D. Toussaint (World Scientific, Singapore, 1990).

[7] M. Luscher and U. Wolff, Nucl. Phys. B 339 (1990) 222.

[8] G. T. Fleming, hep-lat/0403023.

[9] S. R. Beane et al., Phys. Rev. D 79, 114502 (2009) arXiv:0903.2990 [hep-lat]].

[10] S. R. Beane, et al., Phys. Rev. D 80, 074501 (2009) arXiv:0905.0466 [hep-lat]].

[11] T. Yamazaki, Y. Kuramashi and A. Ukawa, Phys. Rev. D 84, 054506 (2011) arXiv:1105.1418 [hep-lat]].

[12] N. Ishii, et al. [HAL QCD Collaboration], Phys. Lett. B, in press, arXiv:1203.3642 [hep-lat].

[13] M. Fukugita, Y. Kuramashi, H. Mino, M. Okawa and A. Ukawa, Phys. Rev. Lett. 73, 2176 (1994) hep-lat/9407012.

[14] M. Fukugita, Y. Kuramashi, M. Okawa, H. Mino and A. Ukawa, Phys. Rev. D 52, 3003 (1995) |hep-lat/9501024.

[15] M. Luscher, Commun. Math. Phys. 104, 177 (1986).

[16] M. Luscher, Commun. Math. Phys. 105, 153 (1986).

[17] M. Luscher, Nucl. Phys. B 354, 531 (1991).

[18] S. R. Beane et al. [NPLQCD Collaboration], Phys. Rev. D 85, 054511 (2012) arXiv:1109.2889 [hep-lat]]. 
[19] H. Nemura, N. Ishii, S. Aoki and T. Hatsuda, Phys. Lett. B673, 136 (2009) [arXiv:0806.1094 [nucl-th]].

[20] T. Inoue et al. [HAL QCD Collaboration], Prog. Theor. Phys. 124, 591 (2010) [arXiv:1007.3559 [hep-lat]].

[21] T. Inoue et al. [HAL QCD Collaboration], Phys. Rev. Lett. 106, 162002 (2011) [arXiv:1012.5928 [hep-lat]].

[22] K. Murano, N. Ishii, S. Aoki and T. Hatsuda, Prog. Theor. Phys. 125, 1225 (2011) arXiv:1103.0619 [hep-lat]].

[23] S. Aoki et al. [HAL QCD Collaboration], Proc. Japan Acad. B 87, 509 (2011) arXiv:1106.2281 [hep-lat]].

[24] T. Inoue et al. [HAL QCD Collaboration], Nucl. Phys. A 881, 28 (2012) arXiv:1112.5926 [hep-lat].

[25] K. Sasaki [HAL QCD Collaboration], PoS LATTICE 2011, 173 (2011).

[26] Y. Ikeda [HAL QCD Collaboration], PoS LATTICE 2011, 159 (2011) arXiv:1111.2663 [hep-lat].

[27] W. Detmold, M. J. Savage, A. Torok, S. R. Beane, T. C. Luu, K. Orginos and A. Parreno, Phys. Rev. D 78, 014507 (2008) arXiv:0803.2728 [heplat]].

[28] J. Foley, K. Jimmy Juge, A. O'Cais, M. Peardon, S. M. Ryan and J. -I. Skullerud, Comput. Phys. Commun. 172, 145 (2005) hep-lat/0505023.

[29] M. Peardon et al. [Hadron Spectrum Collaboration], Phys. Rev. D 80, 054506 (2009) arXiv:0905.2160 [hep-lat]].

[30] W. Detmold and M. J. Savage, Phys. Rev. D 82, 014511 (2010) arXiv:1001.2768 [hep-lat]].

[31] W. Detmold, K. Orginos and Z. Shi, arXiv:1205.4224 [hep-lat].

[32] D. Kaplan, Talk given at Workshop on "Domain Wall Fermions at Ten Years", Mar. 2007, RIKEN BNL Research Center, NY, USA, https://www. bnl.gov/riken/dwf/talks/files/Kaplan_BNL_talk.pdf 